Kragujevac Journal of Mathematics

Volume 39(2) (2015), Pages 255-266.

\title{
ON STABILITY AND BOUNDEDNESS PROPERTIES OF SOLUTIONS OF CERTAIN SECOND ORDER NON-AUTONOMOUS NONLINEAR ORDINARY DIFFERENTIAL EQUATION
}

\author{
J. G. ALABA ${ }^{1}$ AND B. S. OGUNDARE ${ }^{2}$
}

\begin{abstract}
In this paper, sufficient criteria for the existence of solutions to uniform asymptotic stability and boundedness problems associated with certain second order nonlinear non autonomous ordinary differential equation are established with the aid of Lyapunov's direct method. Furthermore, the appropriate complete Lyapunov function is given explicitly. Our results complement some well known results on the second order differential equations in the literature.
\end{abstract}

\section{INTRODUCTION}

Consider the second order non autonomous damped and forced nonlinear ordinary differential equation of the form

$$
(a(t) \dot{x})^{\prime}+b(t) f(x, \dot{x}) \dot{x}+c(t) g(x)=p(t ; x, \dot{x}),
$$

where $f \in C(\mathbb{R} \times \mathbb{R}, \mathbb{R}), g \in C(\mathbb{R}, \mathbb{R}), p \in C([0, \infty) \times \mathbb{R} \times \mathbb{R}, \mathbb{R}), a(t), b(t)$ and $c(t)$ are positive functions.

The functions $a, b, c, f, g$ and $p$ depend only on the arguments displayed explicitly and they are such that existence, uniqueness and continuous dependence on the initial condition are guaranteed. The equation (1.1) can be written as

$$
a(t) \ddot{x}+[\dot{a}(t)+b(t) f(x, \dot{x})] \dot{x}+c(t) g(x)=p(t ; x, \dot{x})
$$

with an equivalent system,

$$
\begin{aligned}
& \dot{x}=y, \\
& \dot{y}=-\frac{1}{a(t)}[\dot{a}(t) y+b(t) f(x, y) y+c(t) g(x)-p(t ; x, y)] .
\end{aligned}
$$

Key words and phrases. Asymptotic stability, boundedness, Lyapunov function, nonlinear, non autonomous, differential equation.

2010 Mathematics Subject Classification. Primary: 34A34, 34D20, 34D23. Secondary:34D99.

Received: November 4, 2014.

Revised: July 17, 2015. 
On substituting $\frac{1}{a(t)}=d(t)$ for all $a(t) \neq 0$ into system (1.2), we have

$$
\begin{aligned}
& \dot{x}=y, \\
& \dot{y}=-[\dot{a}(t) y+b(t) f(x, y) y+c(t) g(x)-p(t ; x, y)] d(t) .
\end{aligned}
$$

The dots indicate differentiation with respect to the independent variable $t$.

For over six decades, numerous works have been done by various authors on autonomous and non autonomous second order nonlinear ordinary differential equations and obtained many interesting results, for examples see [7,10-12,14] and the references cited therein.

In these aforementioned works, the authors made use of Lyapunov direct's method by constructing different Lyapunov functions and obtained criteria which ensure the existence of solutions of some qualitative properties of solutions of the problems considered while some constructed Lyapunov functions, some adopted existing Lyapunov functions to give suitable criteria that guaranteed their results. For more exposition on Lyapunov functions and the qualitative properties of solutions of ordinary differential equations, readers are referred to $[5,13,17,24,25,35,36]$.

Furthermore, many of these existing Lyapunov functions are either incomplete or contain signum functions.

In 1974, Baker [3] established some sufficient conditions that guarantee the uniform stability of the trivial solutions of the following second order nonlinear differential equation

$$
\left(r(t) u^{\prime}\right)^{\prime}+\phi\left(t, u, u^{\prime}\right) u^{\prime}+p(t) f(u)=0 .
$$

with the equivalent system

$$
\begin{aligned}
& \dot{x}=y, \\
& \dot{y}=-\frac{1}{r(t)}\left[r^{\prime}(t) y+\phi(t, x, y) y+p(t) f(x)\right],
\end{aligned}
$$

where $f: \mathbb{R} \rightarrow \mathbb{R}, \phi: I \times \mathbb{R}^{2} \rightarrow \mathbb{R}$ and $p: I \rightarrow \mathbb{R}$ are continuous, and $r: I \rightarrow \mathbb{R}$ is differentiable, and $r(t)>0$ for all $t \in I, I=[0, \infty]$.

He constructed more than one Lyapunov functions for the system (1.4) and obtained conditions to establish his stability results.

In 2011, Tunc [31] studied and extended the results of Baker [3]. He also considered

$$
\left(r(t) x^{\prime}\right)^{\prime}+\phi\left(t, x, x^{\prime}\right) x^{\prime}+p(t) f(x)=p\left(t, x, x^{\prime}\right),
$$

with an equivalent system given as

$$
\begin{aligned}
& \dot{x}=y, \\
& \dot{y}=-\frac{1}{r(t)}\left[r^{\prime}(t) y+\phi(t, x, y) y+p(t) f(x)\right]+\frac{1}{r(t)} q(t, x, y),
\end{aligned}
$$

where $q: I \times \mathbb{R}^{2} \rightarrow \mathbb{R}$ is continuous. He constructed more than one Lyapunov functions for the system (1.6) and proved boundedness of the solutions. 
Other second order differential equations similar to equations (1.1) and (1.5) have been considered by $[1-6,8,9,15,16,18-23,26-30,32-34]$.

Recently, Alaba and Ogundare [1] discussed the uniform asymptotic stability and boundedness of solutions of the of the equation (1.4) or (1.5) with $r(t)=1$ and $\phi(t, x, y) \equiv a(t) f(x, y)$ i.e.

$$
\ddot{x}+a(t) f(x, \dot{x}) \dot{x}+b(t) g(x)=p(t ; x, \dot{x}),
$$

with the equivalent system

$$
\begin{aligned}
& \dot{x}=y, \\
& \dot{y}=-a(t) f(x, y) y-b(t) g(x)+p(t ; x, y),
\end{aligned}
$$

where $f \in C(\mathbb{R} \times \mathbb{R}, \mathbb{R}), g \in C(\mathbb{R}, \mathbb{R}), p \in C([0, \infty) \times \mathbb{R} \times \mathbb{R}, \mathbb{R}), a(t)$ and $b(t)$ are positive functions.

The functions $a, b, f, g$ and $p$ depend only on the arguments displayed explicitly. In the work, they constructed a single complete Lyapunov function and obtained criteria to establish their results.

The motivation for this present work comes from the papers of Baker [3] and C. Tunc [31]. The purpose of this paper is to extend and improve their obtained results by constructing a single unique complete Lyapunov function with less restrictive conditions.

\section{The Main Results}

2.1. Basic Assumptions. The following are the basic assumptions used to formulate the main results of this article.

Let $a(t), b(t), c(t), d(t), f, g$ and $p$ be continuous functions, $I=[0, a b]$ the RouthHurwitz interval, also $a, b, c$ and $d$ are positive i.e, $a, b, c, d>0$ with $0<a_{0} \leq a \leq$ $a(t) \leq a_{1}, 0<b_{0} \leq b \leq b(t) \leq b_{1}, 0<c_{0} \leq c \leq c(t) \leq c_{1}$ and $0<d_{0}<d \leq d(t) \leq d_{1}$, for all $t \in \mathbb{R}^{+}$; in addition, $a, b, c$ and $d$ are differentiable with $a(t), b(t), c(t)$ and $d(t)$ being decreasing functions i.e, $\dot{a} \leq 0, \dot{b} \leq 0, \dot{c} \leq 0$ and $\dot{d} \leq 0$, then:

(i) $|f(x, y)| \leq \alpha \in I$;

(ii) $G_{0}=\frac{g(x)-g(0)}{x} \leq \beta \in I$, with $x \neq 0, g(0)=0$ and $\alpha, \beta>0$;

(iii) $c\left[c \dot{d} \alpha^{2}+\beta\left(2 b d \alpha+\frac{\dot{b}}{b}(b+1)\right)\right]>\dot{c} \beta(\delta+1)+b \alpha^{2}(2 b \dot{d}+\dot{b} d)$ for all $\alpha, \beta, \delta$ all positive;

(iv) $p(t ; x, y)=p(t)$ and $|p(t)| \leq W$ for all $t \leq 0$, where $W$ is a constant.

These are the main results with respect to the equation (1.1).

Theorem 2.1. Suppose conditions (i)-(iii) in our basic assumptions hold, then the trivial solution of the system (1.3) is globally asymptotically stable.

Theorem 2.2. Suppose that the conditions of Theorem 2.1 hold and in addition condition (iv) of the basic assumption also holds, then there exists $\sigma(0<\sigma<\infty)$ 
depending only on $\alpha, \beta$ and $\delta$ such that every solution of system (1.3) satisfies

$$
x^{2}(t)+\dot{x}^{2}(t) \leq e^{-\sigma t}\left\{Q_{1}+Q_{2} \int_{t_{0}}^{t}|p(\tau)| e^{\left(\frac{\sigma \tau}{2}\right)} d \tau\right\}^{2},
$$

for all $t \geq t_{0}$, where the constant $Q_{1}>0$ depends on $\alpha, \beta, \delta, t_{0}, x\left(t_{0}\right), \dot{x}(t)$ and the constant $Q_{2}>0$ depends on $\alpha, \beta$ and $\delta$ only.

Theorem 2.3. Suppose the conditions of Theorem 2.2 hold with condition (iv) replaced with $(v)|p(t ; x, y)| \leq(|x|+|y|) \phi(t)$, where $\phi(t)$ is a non-negative and continuous function of $t$ and satisfies $\int_{0}^{t} \phi(s) d s \leq W<\infty, W>0$ is a constant. Then there exists a constant $\lambda_{0}$ which depends on $W, \lambda_{1}, \lambda_{2}$ and $t_{0}$ such that every solution $x(t)$ of system (1.3) satisfies $|x(t)| \leq \lambda_{0},|\dot{x}(t)| \leq \lambda_{0}$, for sufficiently large $t$.

\section{Preliminary Results}

The main tool employed in proving our main results is the scalar function $V(t ; x, y)$ defined as

$$
V(t ; x, y)=\frac{1}{2 b d \alpha}\left\{d c \beta(\delta+1) x^{2}+(d b \alpha)^{2} x^{2}+\delta y^{2}+y^{2}+2 b d \alpha x y\right\} .
$$

To establish that (3.1) is indeed a Lyapunov function for the system (1.3), we shall state and prove the following Lemmas.

Lemma 3.1. Subject to the assumptions of Theorem 2.1, there exist positive constants $\lambda_{i}=\lambda_{i}(a, b, c, d, \alpha, \beta, \delta) i=1,2$ such that

$$
\lambda_{1}\left(x^{2}+y^{2}\right) \leq V(t ; x, y) \leq \lambda_{2}\left(x^{2}+y^{2}\right)
$$

Proof. Clearly, $V(t ; 0,0)=0$. By rewriting (3.1) we have

$$
\begin{aligned}
& V=\frac{1}{2 b d \alpha}\left\{d c \beta(\delta+1) x^{2}+\delta y^{2}+(b d \alpha x+y)^{2}\right\}, \\
& V \geq \frac{1}{2 b d \alpha}\left\{d c \beta(\delta+1) x^{2}+\delta y^{2}\right\}
\end{aligned}
$$

and

$$
V(t ; x, y) \geq \lambda_{1}\left(x^{2}+y^{2}\right)
$$

where $\lambda_{1}=\lambda_{0} \times \min \left\{d_{0} c_{0} \beta(\delta+1), \delta\right\}$ and $\lambda_{0}=\frac{1}{2 d b \alpha}$.

After applying the inequality $|x y| \leq \frac{1}{2}\left|x^{2}+y^{2}\right|$ on equation (3.1), we have

$$
\begin{aligned}
& V \leq \frac{1}{2 b d \alpha}\left\{\left[d c \beta(\delta+1)+(b d \alpha)^{2}\right] x^{2}+[\delta+1] y^{2}+b d \alpha x^{2}+b d \alpha y^{2}\right\}, \\
& V \leq \frac{1}{2 b d \alpha}\left\{\left[d c \beta(\delta+1)+(b d \alpha)^{2}+b d \alpha\right] x^{2}+[b d \alpha+\delta+1] y^{2}\right\},
\end{aligned}
$$


and

$$
V(t ; x, y) \leq \lambda_{2}\left(x^{2}+y^{2}\right)
$$

where $\lambda_{2}=\lambda_{0} \times \max \left\{d_{1} c_{1} \beta(\delta+1)+\left(d_{1} b_{1} \alpha\right)^{2}+b_{1} d_{1} \alpha, b_{1} d_{1} \alpha+\delta+1\right\}$ and $\lambda_{0}=\frac{1}{2 d b \alpha}$. From equations (3.4) and (3.7), we have

$$
\lambda_{1}\left(x^{2}+y^{2}\right) \leq V(t ; x, y) \leq \lambda_{2}\left(x^{2}+y^{2}\right)
$$

which completes the proof.

Lemma 3.2. Subject to the assumptions of Theorem 2.1, there exist positive constants $\lambda_{j}=\lambda_{j}(a, b, c, d, \alpha, \beta, \delta)$ with $j=3,4$ such that for any solution $(x, y)$ of system (1.3)

$$
\left.\dot{V}(t ; x, y)\right|_{(1.4)}=\left.\frac{d V(t ; x, y)}{d t}\right|_{(1.4)} \leq-\lambda_{3}\left(x^{2}+y^{2}\right)+\lambda_{4}(|x|+|y|)|p(t ; x, y)| .
$$

Proof. After differentiating the equation (3.1) with respect to $t$, we have

$$
\left.\frac{d V}{d t}\right|_{(1.4)}=\left.\dot{V}\right|_{(1.4)}=\frac{\partial V}{\partial t}+\frac{\partial V}{\partial x} \dot{x}+\frac{\partial V}{\partial y} \dot{y}
$$

by using (1.3) in (3.9). Therefore, differentiation of (3.1) partially, with respect to $t$, gives

$$
\begin{aligned}
\frac{\partial V}{\partial t}= & \frac{2 b d \alpha}{4 b^{2} d^{2} \alpha^{2}}\left\{\left[d \dot{c} \beta(\delta+1)+\dot{d} c \beta(\delta+1)+2 \dot{d} d b^{2} \alpha^{2}+2 \dot{b} b d^{2} \alpha^{2}\right] x^{2}+2(\dot{b} d+b \dot{d}) \alpha x y\right. \\
& \left.-\frac{2 \alpha(\dot{b} d+b \dot{d})}{4 b^{2} d^{2} \alpha^{2}}\left(\left[d c \beta(\delta+1)+(d c \alpha)^{2}\right] x^{2}+[\delta+1] y^{2}+2 b d \alpha x y\right)\right\} \\
= & \frac{1}{2 b d \alpha}\left\{\left(\left[d \dot{c} \beta(\delta+1)+\dot{d} c \beta(\delta+1)+2 \dot{d} d b^{2} \alpha^{2}+2 \dot{b} b d^{2} \alpha^{2}\right] x^{2}+2(\dot{b} d+b \dot{d}) \alpha x y\right)\right. \\
& -\frac{1}{2 b^{2} d^{2} \alpha}\left(\left[\dot{d} d^{2} c \beta(\delta+1)+\dot{b} d(d b \alpha)^{2}+b \dot{d} d c \beta(\delta+1)+b \dot{d} d^{2} c^{2} \alpha^{2}\right] x^{2}\right. \\
& \left.\left.+[\dot{b} d(\delta+1)+b \dot{d}(\delta+1)] y^{2}+2 \dot{b} b d^{2} \alpha x y+2 b^{2} \dot{d} d \alpha x y\right)\right\} .
\end{aligned}
$$

Further simplification yields to

$$
\begin{aligned}
\frac{\partial V}{\partial t}= & \frac{1}{b d \alpha}\left\{\frac{1}{2}\left[d \dot{c} \beta(\delta+1)+2 \dot{d} d b^{2} \alpha^{2}+\dot{b} b d^{2} \alpha^{2}-\frac{\dot{b}}{b}(\delta+1)-\dot{d} d c^{2} \alpha^{2}\right] x^{2}\right. \\
& \left.-\frac{1}{2}\left[\dot{b} b(\delta+1)+\frac{\dot{d}}{d}(\delta+1)\right] y^{2}\right\}
\end{aligned}
$$


Also differentiating the equation (3.1) partially with respect to $x$ gives

$$
\begin{aligned}
\frac{\partial V}{\partial x} & =\frac{1}{d b \alpha}\left\{\left[d c \beta(\delta+1)+(d b \alpha)^{2}\right] x+d b \alpha y\right\}, \\
\frac{\partial V}{\partial x} \dot{x} & =\frac{1}{d b \alpha}\left\{\left[d c \beta(\delta+1)+(d b \alpha)^{2}\right] x y+d b \alpha y^{2}\right\} .
\end{aligned}
$$

Finally differentiating the equation (3.1) partially with respect to $y$ gives

$$
\begin{aligned}
\frac{\partial V}{\partial y} & =\frac{1}{d b \alpha}\{(\delta+1) y+d b \alpha x\}, \\
\frac{\partial V}{\partial y} \dot{y}= & \frac{1}{d b \alpha}\{((\delta+1) y+d b \alpha x)(-\dot{a} d y-b d f(x, y)-c d g(x)+d p(t ; x, y))\} \\
\leq & \frac{1}{b d \alpha}\left\{-b c d^{2} \alpha \beta x^{2}-\dot{a} d(\delta+1) y^{2}-b d \alpha(\delta+1) y^{2}-b^{2} d^{2} \alpha^{2} x y-c d \beta(\delta+1) x y\right. \\
& \left.+p(t ; x, y) d^{2} b \alpha x+p(t ; x, y)(\delta+1) d y\right\},
\end{aligned}
$$

and

$$
\begin{aligned}
\frac{\partial V}{\partial y} \dot{y}= & \frac{1}{b d \alpha}\left\{\left(-b c d^{2} \alpha \beta x^{2}-[\dot{a} d(\delta+1)+b d \alpha(\delta+1)]\right) y^{2}\right. \\
& \left.-\left[b^{2} d^{2} \alpha^{2}+c d \beta(\delta+1)\right] x y+p(t ; x, y) d^{2} b \alpha x+p(t ; x, y)(\delta+1) d y\right\}
\end{aligned}
$$

If we substitute equations (3.10), (3.11) and (3.12) in (3.9) then it yields to

$$
\begin{aligned}
\frac{d V}{d t}= & -\frac{1}{b d \alpha}\left\{\left(b c d^{2} \alpha \beta+\frac{1}{2}\left[\dot{d} d c^{2} \alpha^{2}+\frac{\dot{b}}{b} d c \beta(\delta+1)\right]\right.\right. \\
& \left.-\frac{1}{2}\left[d \dot{c} \beta(\delta+1)+2 \dot{d} d b^{2} \alpha^{2}+\dot{b} b d^{2} \alpha^{2}\right]\right) x^{2} \\
+ & \left(\dot{a} d(\delta+1)+b d \alpha \delta+\frac{1}{2}\left(\frac{\dot{b}}{b}(\delta+1)+\frac{\dot{d}}{d}(\delta+1)\right)\right) y^{2} \\
& \left.+p(t ; x, y)\left(d^{2} b \alpha x+(\delta+1) d y\right)\right\}
\end{aligned}
$$

and

$$
\frac{d V}{d t}=\dot{V}(t ; x, y) \leq-\lambda_{3}\left(x^{2}+y^{2}\right)+\lambda_{*}(|x|+|y|) p(t ; x, y)
$$


with $\lambda_{0}=\frac{1}{d b \alpha}, \lambda_{*}=\left\{d^{2} b \alpha, d(\delta+1)\right\}$ and

$$
\begin{aligned}
\lambda_{3}= & \lambda_{0} \times \max \left\{b c d^{2} \alpha \beta+\frac{1}{2}\left[\dot{d} d c^{2} \alpha^{2}+\frac{\dot{b}}{b} d c \beta(\delta+1)\right]-\frac{1}{2}\left[d \dot{c} \beta(\delta+1)+2 \dot{d} d b^{2} \alpha^{2}\right.\right. \\
& \left.\left.+\dot{b} b d^{2} \alpha^{2}\right],\left[\dot{a} d(\delta+1)+b d \alpha \delta+\frac{1}{2}\left(\frac{\dot{b}}{b}(\delta+1)+\frac{\dot{d}}{d}(\delta+1)\right)\right]\right\} .
\end{aligned}
$$

Inequality (3.14) can also be simplified and given as

$$
\frac{d V}{d t}=\dot{V}(t ; x, y) \leq-\lambda_{3}\left(x^{2}+y^{2}\right)+\lambda_{4}\left(x^{2}+y^{2}\right)^{\frac{1}{2}}|p(t ; x, y)|
$$

with $\lambda_{4}=\sqrt{2} \lambda_{*}$ which completes the proof.

Remark 3.1. When $p(t ; x, y)=0,(3.15)$ becomes

$$
\frac{d V}{d t}=\dot{V}(t ; x, y) \leq-\lambda_{3}\left(x^{2}+y^{2}\right) .
$$

Lemma 3.1 established that $V$ is a positive definite function while Lemma 3.2 showed that $\dot{V}$ is negative definite function; hence $V$ is a Lyapunov function for the system (1.3).

\section{Proof of the Main Results}

Now, we shall give the proofs of our results stated in section 4 as follows.

Proof of Theorem 2.1. From Lemmas 3.1 and 3.2 with $p(t ; x, \dot{x}) \equiv 0$, it had been established that function $V(t ; x, y)$ is a Lyapunov function for the system (1.3). Hence, the trivial solution of system (1.3) is globally asymptotically stable (g.a.s), that is, every solution $x(t), \dot{x}(t)$ of the system (1.3) satisfies $x^{2}(t)+\dot{x}^{2}(t) \rightarrow 0$ as $t \rightarrow \infty$.

Proof of Theorem 2.2. From (3.15) by replacing $p(t ; x, \dot{x})$ with $p(t)$ we obtain

$$
\dot{V}(t ; x, y) \leq-\lambda_{3}\left(x^{2}+y^{2}\right)+\lambda_{4}\left(x^{2}+y^{2}\right)^{\frac{1}{2}} p(t) .
$$

From (3.4), we have

$$
x^{2}+y^{2} \leq \frac{1}{\lambda_{1}} V
$$

Hence

$$
\lambda_{3}\left(x^{2}+y^{2}\right) \leq \lambda_{3} \frac{V}{\lambda_{1}} .
$$

Using inequalities (4.2) and (4.3), inequality (4.1) becomes

$$
\frac{d V}{d t} \leq-\lambda_{6} V+\lambda_{5} V^{\frac{1}{2}}|p(t)|
$$


where $\lambda_{6}=\frac{\lambda_{3}}{\lambda_{1}}$ and $\lambda_{5}=\frac{\lambda_{4}}{\lambda_{1}^{\frac{1}{2}}}$. The inequality (4.4) can also be expressed as

$$
\frac{d V}{d t} \leq-2 \lambda_{7} V+\lambda_{5} V^{\frac{1}{2}}|p(t)|
$$

with $\lambda_{7}=\frac{\lambda_{6}}{2}$. Therefore,

$$
\begin{aligned}
& \dot{V}+\lambda_{7} V \leq-\lambda_{7} V+\lambda_{5} V^{\frac{1}{2}}|p(t)|, \\
& \dot{V}+\lambda_{7} V \leq \lambda_{5} V^{\frac{1}{2}}\left\{|p(t)|-\lambda_{8} V^{\frac{1}{2}}\right\},
\end{aligned}
$$

where $\lambda_{8}=\frac{\lambda_{7}}{\lambda_{5}}$. Thus (4.5) becomes $\dot{V}+\lambda_{7} V \leq \lambda_{5} V^{\frac{1}{2}} V^{*}$, where

$$
V^{*}=|p(t)|-\lambda_{8} V^{\frac{1}{2}} .
$$

When $|p(t)| \leq \lambda_{8} V^{\frac{1}{2}}$, then (4.6) becomes

$$
V^{*} \leq 0
$$

and when $|p(t)| \geq \lambda_{8} V^{\frac{1}{2}}$, then (4.6) becomes $V^{*} \geq 0$. By substituting (4.7) into (4.5), we have $\dot{V}+\lambda_{7} V \leq \lambda_{9} V^{\frac{1}{2}}|p(t)|$, where $\lambda_{9}=\frac{\lambda_{5}}{\lambda_{8}}$. This implies that

$$
\dot{V} V^{-\frac{1}{2}}+\lambda_{7} V^{\frac{1}{2}} \leq \lambda_{9}|p(t)| \text {. }
$$

Multiplying both sides of (4.8) by $e^{\frac{\lambda_{7} t}{2}}$, we have $e^{\frac{\lambda_{7} t}{2}}\left\{\dot{V} V^{-\frac{1}{2}}+\lambda_{7} V^{\frac{1}{2}}\right\} \leq e^{\frac{\lambda_{7} t}{2}} \lambda_{9}|p(t)|$, that is,

$$
2 \frac{d}{d t}\left\{V^{\frac{1}{2}} e^{\left(\frac{\lambda_{7} t}{2}\right)}\right\} \leq e^{\frac{\lambda_{7} t}{2}} \lambda_{9}|p(t)| .
$$

Integrating both sides of (4.9) from $t_{0}$ to $t$, gives

$$
\left\{V^{\frac{1}{2}} e^{\frac{\lambda_{7} \xi}{2}}\right\}_{t_{0}}^{t} \leq \int_{t_{0}}^{t} \frac{1}{2} e^{\frac{\lambda_{7} \tau}{2}} \lambda_{9}|p(\tau)| d \tau
$$

which implies that

$$
\left\{V^{\frac{1}{2}}(t)\right\} e^{\left(\frac{\lambda_{7} t}{2}\right)} \leq V^{\frac{1}{2}}\left(t_{0}\right) e^{\frac{\lambda_{7} t_{0}}{2}}+\frac{\lambda_{9}}{2} \int_{t_{0}}^{t}|p(\tau)| e^{\frac{\lambda_{7} \tau}{2}} d \tau
$$

or

$$
V^{\frac{1}{2}}(t) \leq e^{-\left(\frac{\lambda_{7} t}{2}\right)}\left\{V^{\frac{1}{2}}\left(t_{0}\right) e^{\frac{\lambda_{7} t_{0}}{2}}+\frac{\lambda_{9}}{2} \int_{t_{0}}^{t}|p(\tau)| e^{\frac{\lambda_{7} \tau}{2}} d \tau\right\} .
$$

Using (3.4) and (4.10), we have

$$
\lambda_{1}\left(x^{2}(t)+\dot{x}^{2}(t)\right) \leq e^{-\left(\lambda_{7} t\right)}\left\{\lambda_{2}\left(x(t)^{2}+\dot{x}^{2}(t)\right)^{\frac{1}{2}} e^{\frac{\lambda_{7} t_{0}}{2}}+\frac{\lambda_{9}}{2} \int_{t_{0}}^{t}|p(\tau)| e^{\frac{\lambda_{7} \tau}{2}} d \tau\right\}^{2},
$$


for all $t \geq 0$. Thus

$$
\begin{aligned}
x^{2}(t)+\dot{x}^{2}(t) & \leq \frac{1}{\lambda_{1}}\left\{e^{-\lambda_{7} t}\left\{\lambda_{2}\left(x^{2}(t)+\dot{x}^{2}(t)\right)^{\frac{1}{2}} e^{\frac{\lambda_{7} t_{0}}{2}}+\frac{\lambda_{9}}{2} \int_{t_{0}}^{t}|p(\tau)| e^{\frac{\lambda_{7} \tau}{2}} d \tau\right\}^{2}\right\} \\
& \leq e^{-\lambda_{7} t}\left\{Q_{1}+Q_{2} \int_{t_{0}}^{t}|p(\tau)| e^{\frac{\lambda_{7} \tau}{2}} d \tau\right\}^{2},
\end{aligned}
$$

where $Q_{1}=\lambda_{2}\left(x(t)^{2}+\dot{x}^{2}(t)\right)^{\frac{1}{2}} e^{\frac{\lambda_{7} t_{0}}{2}}$ and $Q_{2}=\frac{\lambda_{9}}{2}$.

By putting $\lambda_{7}=\sigma$ in the inequality (4.11) and gives

$$
x^{2}(t)+\dot{x}^{2}(t) \leq e^{-\sigma t}\left\{Q_{1}+Q_{2} \int_{t_{0}}^{t}|p(\tau)| e^{\left(\frac{\sigma \tau}{2}\right)} d \tau\right\}^{2} .
$$

Hence, this completes the proof.

Remark 4.1. From the proof of the Theorem 2.2, the following can be pointed out as direct consequence of the Theorem. If $p(t, x, y) \equiv 0$, then the trivial solution of (1.3) is uniformly asymptotically stable.

Remark 4.2. If $p(t ; x, y) \equiv 0$, then (4.12) reduces to $x^{2}(t)+\dot{x}^{2}(t) \leq e^{-\sigma t} Q_{1}$, as $t \rightarrow \infty$, $x^{2}(t)+\dot{x}^{2}(t) \rightarrow 0$ which implies that the trivial solution of the system (1.3) is globally asymptotically stable.

Proof of Theorem 2.3. Indeed from the inequality (3.15), we have

$$
\dot{V} \leq \lambda_{*}(|x|+|y|)^{2} \phi(t) \text {. }
$$

By using the Schwartz inequalities on (4.13) we obtain

$$
\dot{V} \leq \lambda_{10}\left(x^{2}+y^{2}\right) \phi(t)
$$

where $\lambda_{10}=2 \lambda_{*}$.

From the inequalities (3.4) and (4.10), we have $\dot{V} \leq \lambda_{10} V \phi(t)$, by integrating equation (4.14) from 0 to $t_{0}$, we obtain

$$
V(t)-V(0) \leq \lambda_{11} \int_{t_{0}}^{t} V(s) \phi(s) d s,
$$

where $\lambda_{11}=\frac{\lambda_{10}}{\lambda_{1}}=\frac{3 \lambda_{*}}{\lambda_{1}}$. The inequality (4.15) now becomes

$$
V(t) \leq V(0)+\lambda_{11} \int_{t_{0}}^{t} V(s) \phi(s) d s .
$$

By Gronwall-Bellman inequality (4.16) we obtain

$$
V(t) \leq V(0) \exp \left(\lambda_{11} \int_{t_{0}}^{t} \phi(s) d s\right)
$$

This completes the proof. 


\section{EXAMPLE}

Consider second order non autonomous nonlinear differential equation

$$
\left(\frac{2+3 t^{2}}{1+t^{2}} x^{\prime}\right)^{\prime}+\frac{t}{\left(1+t^{2}\right)^{2}} x^{\prime}+\frac{4+3 t^{2}}{1+t^{2}}\left(2 x+4 x^{3}\right)=\frac{1}{1+t^{2}+x^{2}+x^{\prime 2}},
$$

which can be written as

$$
\left(3-\frac{1}{1+t^{2}}\right) x^{\prime \prime}+\frac{3 t}{\left(1+t^{2}\right)^{2}} x^{\prime}+\frac{4+3 t^{2}}{1+t^{2}}\left(2 x+4 x^{3}\right)=\frac{1}{1+t^{2}+x^{2}+x^{\prime 2}} .
$$

We state (5.2) as the system form

$$
\begin{aligned}
& x^{\prime}=y, \\
& y^{\prime}=-\frac{3 t}{\left(2+3 t^{2}\right)\left(1+t^{2}\right)} y-\frac{4+3 t^{2}}{3 t^{2}+2}\left(2 x+4 x^{3}\right)+\frac{1+t^{2}}{\left(1+t^{2}+x^{2}+y^{2}\right)\left(2+3 t^{2}\right)} .
\end{aligned}
$$

Comparing (5.1) with (1.1), it is clearly seen that

$$
\begin{aligned}
& a(t)=3-\frac{1}{1+t^{2}}, \quad t \geq 0, \quad 2 \leq 3-\frac{1}{1+t^{2}} \leq 3, \\
& a_{0}=2, \quad a_{1}=3, \\
& b(t)=\frac{t}{\left(1+t^{2}\right)^{2}}, \quad t \geq 0, \quad \frac{1}{2} \leq \frac{t}{\left(1+t^{2}\right)^{2}} \leq 1, \\
& b_{0}=\frac{1}{4}, \quad b_{1}=\frac{2}{5}, \\
& c(t)=3+\frac{1}{1+t^{2}}, \quad t>0, \quad 3 \leq 3+\frac{1}{1+t^{2}} \leq 4, \\
& c_{0}=3, \quad c_{1}=4, \\
& d_{0}=\frac{1}{2}, \quad d_{1}=\frac{1}{3}, \\
& \lambda_{0}=\frac{1}{\alpha}, \quad \lambda_{3}=12 \beta .
\end{aligned}
$$

The corresponding Lyapunov function to the system (5.3) is given as

$$
V=\frac{1}{\alpha}\left\{0.5 \beta(\delta+1) x^{2}+\delta y^{2}+(0.5 \alpha x+y)^{2}\right\}>0,
$$

where $\alpha, \beta$, and $\delta$ are positive constants and whose derivative is given as

$$
\frac{d V}{d t}=\dot{V}(t ; x, y) \leq-12 \beta\left(x^{2}+y^{2}\right)
$$

where $\beta>0$. All conditions stated in Theorem 2.1 are satisfied therefore the zero solution of system (5.3) is globally asymptotic stable.

We have for $p \equiv 0$ that the solutions of (5.1) are globally asymptotic stable. 
Acknowledgments. The authors wish to acknowledge the efforts of the anonymous referees for their constructive criticism and useful suggestions that improved the quality of this work.

\section{REFERENCES}

[1] J. G. Alaba and B. S. Ogundare, Asymptotic behaviour of solutions of certain second order non-autonomous nonlinear ordinary differential equation, Int. J. Pure Appl. Math. 90 (2014), 469-483.

[2] F. V. Atkinson, On second order nonlinear oscillations, Pacific J. Math. 5 (1955), 643-647.

[3] J. W. Baker, Stability properties of a second order damped and forced nonlinear differential equation, SIAM J. Appl. Math. 27(1) (1974), 159-166.

[4] I. Bihari, On periodic solutions of certain second order ordinary differential equations with periodic coefficients, Acta. Math. Acad. Sci. Hungar. 12 (1961), 11-16.

[5] T. A. Burton, Lyapunov functions and boundedness, J. Math. Anal. Appl. 58 (1977), 88-97.

[6] M. L. Cartwright and J. E. Littlewood, On nonlinear differential equations of the second order: $\ddot{y}+k f(y, \dot{y}+g(y, k))=p(t)=p_{1}(t)+k p_{2}(t), k>0, f(y) \geq 1$, Annals of Math. 48(2) (1947), 472-494.

[7] W. J. Coles, Boundedness of solutions of two-dimensional first order differential systems, Boll. Univ. Mat. Ital. 4 (1971), 225-231.

[8] L. Hatvani, On the stability of the zero solution of nonlinear second order differential equations, Acta Sci. Math. (Szeged) 32 (1971), 1-9.

[9] L. Hatvani, On stability properties of solutions of second order differential equations, Electron. J. Qual. Theory Differ. Equ. Proceedings of the 6th Coll. Qualitative Theory of Diff. Equ., 11 (1999), 1-6.

[10] J. JR. Jones, On the asymptotic stability of certain second order nonlinear differential equations, SIAM J. Appl. Math. 14 (1966), 16-22.

[11] J. JR. Jones, On the stability of certain second order nonlinear differential equations, Portugal Math. 29 (1970), 82-86.

[12] A. G. Kartsatos, On oscillation and boundedness of solutions of second order nonlinear differential equations, Boll. Uni. Mat. Ital. 3 (1968), 357-361.

[13] G. Lados, On oscillation and boundedness of solutions of nonlinear differential equations, Boll. Soc. Mat. Greece 10 (1969), 48-54.

[14] G. G. Legatos and A. G. Kartsatos, On the uniform boundedness of solutions of certain nonlinear ordinary differential equations of second order, Boll. Soc. Mat. Greece 6 (1965), 51-58.

[15] Y. S. Lim and L. F. Kazda, A study of second order nonlinear systems, J. Math. Anal. Appl. 8 (1964), 423-444.

[16] W. S. Loud, Boundedness and convergence of solutions of $\ddot{x}+c \dot{x}+g(x)=\exp (t)$, Duke Math. J. 24 (1957), 63-72.

[17] A. M. Lyapunov, The general problem of the stability of motion (in Russian) [Translated and Reproduced in Ann. Math. Study 17 (1947) Princeton, New Jersey.

[18] J. E. Napoles Valdes, A note on the asymptotic stability in the whole of non autonomous systems, Rev. Colombiana Mat. 33 (1999), 1-8.

[19] J. E. Napoles Valdes, A note on the qualitative behavior of some second order nonlinear equation, Appl. Appl. Math. 8 (2013), 767-776.

[20] B. S. Ogundare and G. E. Okecha, Boundedness, periodicity and stability of solutions to $\ddot{x}+$ $a(t) g(\dot{x})+b(t) h(x)=p(t ; x, \dot{x})$, Math. Sci. Res. J. 11 (2007), 432-443.

[21] B. S. Ogundare, S. Ngcibi and V. Murali, Boundedness and stability properties of solutions to certain second order differential equation, Adv. Differ. Equ. Control Process. 5(2) (2010), 79-92. 
[22] P. Pucci and J. Serrin, Precise damping conditions for global asymptotic stability for nonlinear second order systems, Acta. Math. 170 (1993), 275-307.

[23] P. Pucci and J. Serrin, Precise damping conditions for global asymptotic stability for nonlinear second order systems, ll, J. Differential Equations 113 (1994), 505-534.

[24] R. Reissig, G. Sansone and R. Conti Non Linear Differential Equations of Higher Order, Nourdhoff International Publishing Lyden, 1974.

[25] N. Rouche, P. Habets and M. Laloy, Stability Theory by Liapunov's Second Method, SpringVerlag, 1977.

[26] H. O. Tejumola, Boundedness Criteria for solutions of some second-order differential equations, Atti. Accad. Naz. Lincei Rend. Cl. Sci. Fis. Math. Natur. 50 (1971), 432-437.

[27] C. Tunc, A note on boundedness of solutions to a class of a non-autonomous differential equation of second order, Appl. Anal. Discrete Math. 4 (2010), 361-372.

[28] C. Tunc, Boundedness results for solutions of certain nonlinear differential equation of second order, J. Indones. Math. Soc. 16 (2010), 115-126.

[29] C. Tunc, Uniformly stability and boundedness of solutions of second order nonlinear delay differential equations, Appl. Comput. Math. 10 (2011), 449-462.

[30] C. Tunc, Stability and boundedness of solutions of a non-autonomous differential equation of second order, J. Comput. Anal. Appl. 13 (2011), 1067-1074.

[31] C. Tunc, On the Boundedness of solutions of a non-autonomous differential equation of second order, Sarajevo Jour. of Math. 17 (2011), 19-29.

[32] C. Tunc and E. Tunc, On the asymptotic behaviour of solutions of certain second order differential equations, J. Franklin Inst. 344 (2007), 391-398.

[33] C. Tunc and O. Tunc, A Note on certain qualitative properties of a second order linear system, Appl. Math. Inf. Sci. 9 (2015), 953-956.

[34] J. S. W. Wong and T. A. Burton, Some properties of solutions of second order nonlinear non autonomous differential equation, Monnatshefte Fur Mathematik 69 (1965), 368-374.

[35] T. Yoshizawa, Stability Theory by Lyapunov's Second Method, Math. Soc. Japan, 1966.

[36] V. I. Zubov. The methods of Lyapunov and their applications, Noordhoff, Groning, 1964.

${ }^{1}$ Department of Mathematics, OBAFEMi AWOlOWo University, ILE-IFE, 220005, NigERIA

E-mail address: alabajames@yahoo.com

${ }^{2}$ Department of Mathematics, OBAFEmi Awolowo University, Ile-IfE, 220005, Nigeria

E-mail address: ogundareb@yahoo.com, bogunda@oauife.edu.ng 